\title{
INSTABILITY OF THE NON-ISOTHERMAL INCLINED FILM FLOW OF A POWER-LAW FLUID WITH TEMPERATURE DEPENDENT CONSISTENCY
}

\author{
by \\ Mohammad Mahmud Hasan \\ Master of Science, University of Chittagong, 2006 \\ Bachelor of Science, University of Chittagong, 2005 \\ A thesis \\ presented to Ryerson University \\ in partial fulfillment of the \\ requirements for the degree of \\ Master of Science, \\ in the Program of \\ Applied Mathematics \\ Toronto, Ontario, Canada, 2017 \\ (C)Mohammad Mahmud Hasan 2017
}




\section{Author's Declaration}

I hereby declare that I am the sole author of this thesis. This is a true copy of the thesis, including any required final revisions, as accepted by my examiners.

I authorize Ryerson University to lend this thesis to other institutions or individuals for the purpose of scholarly research.

I further authorize Ryerson University to reproduce this thesis by photocopying or by other means, in total or in part, at the request of other institutions or individuals for the purpose of scholarly research.

I understand that my thesis may be made electronically available to the public. 
Instability of the non-isothermal inclined film flow of a power-law fluid with temperature dependent

\author{
consistency \\ Master of Science, 2017 \\ Mohammad Mahmud Hasan \\ Applied Mathematics \\ Ryerson University
}

In this thesis we undertake a theoretical study of the flow stability of a liquid film with power-law rheology down a heated incline. We develop and implement a mathematical model for the flow that captures the variation with temperature of the rheological aspect of the fluid. We carry out a linear stability analysis and obtain Orr-Sommerfeld type equations for the evolution of infintesimal perturbations imposed on the equilibrium flow. We obtain asymptotic solutions based on the assumption of perturbations of long wavelength and small variation in viscosity with respect to temperature. We investigate the critical conditions for the onset of instability and determine the effect of a non-Newtonian reheology and the dependence of the fluid properties on temperature. 


\section{Acknowledgements}

Firstly, I would like to express my sincere gratitude to my supervisor Prof. Dr. Jean-Paul Pascal for the continuous support of my master's study and related research, for his patience, motivation, and immense knowledge. His guidance, encouragement and valuable support helped me in the research and writing of this thesis. I could not have imagined having a better advisor and mentor for my M.Sc study. I owe a debt of gratitude to him for his valuable time and knowledge.

Besides my advisor, I would like to gratefully thank the rest of my thesis committee members: Dr. Katrin Rohlf, Dr. Kathleen Wilkie and Dr. Dejan Delic for their insightful comments, encouragement and valuable suggestions.

In a special way, I would like to thank Ryerson University for offering me the special chance to study in the Applied Mathematics program. I would also like to thank Dr. Sebastian Ferrando and Dr. Silvana Ilie for their guidance as chair of the graduate studies and Dr. Konstantinos Georgiou for organizing the graduate seminars. I also acknowledge all my fellow classmate for their cooperation for the last two years.

Finally, I must express my very profound gratitude to my family: my parents and siblings for providing me with unfailing support and continuous encouragement throughout my years of study and

through the process of researching and writing this thesis. This accomplishment would not have been possible without them. 


\section{Dedication}

I would like to dedicate this thesis to my parents and my supervisor Dr. Jean-Paul Pascal. 


\section{Contents}

Author's Declaration $\quad$ ii

$\begin{array}{ll}\text { Abstract } & \text { iii }\end{array}$

Acknowledgements $\quad$ iv

Dedication $\quad$ v

List of Figures $\quad$ vii

1 Introduction $\quad 1$

2 Governing Equations $\quad 4$

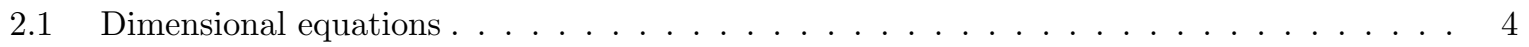

2.2 Non-Dimensional equations $\ldots \ldots \ldots \ldots \ldots \ldots \ldots$

2.3 The long-wave approximation $\ldots \ldots \ldots \ldots \ldots \ldots \ldots$

3 Linear Stability Analysis $\quad 12$

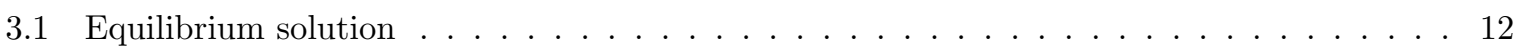

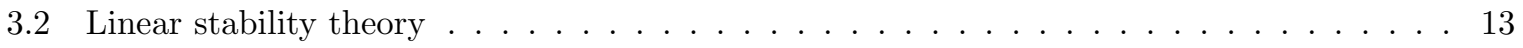

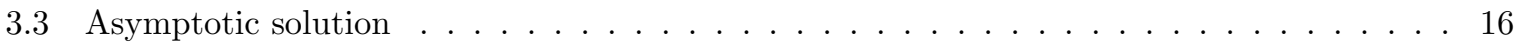

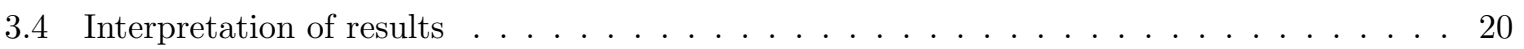

4 Conclusions $\quad 27$

$\begin{array}{lr}\text { References } & 29\end{array}$ 


\section{List of Figures}

2.1 Schematic representation of a thin film flowing down an inclined plane. . . . . . . . . . . 4

$3.1 R e_{\text {crit }}$ as a function of $M a$ for $\cot \theta=1, \operatorname{Pr}=7, B i=1, n=1 \ldots \ldots \ldots \ldots 22$

$3.2 R e_{\text {crit }}$ as a function of $M a$ for $\cot \theta=1, \operatorname{Pr}=7, B i=1, n=0.5 \ldots \ldots \ldots \ldots$

$3.3 R e_{\text {crit }}$ as a function of $M a$ for $\cot \theta=1, \operatorname{Pr}=7, B i=1, \lambda=0.2 \ldots \ldots \ldots 24$

$3.4 R e_{\text {crit }}$ as a function of $M a$ for $\cot \theta=1, \operatorname{Pr}=7, \lambda=0, n=1 \ldots \ldots \ldots \ldots$

$3.5 R e_{\text {crit }}$ for the $\mathrm{H}$ mode as a function of $B i$ for $\cot \theta=1, \operatorname{Pr}=7, M a=0.1, n=1 \ldots \ldots 25$

3.6 Re $e_{\text {crit }}$ for the $\mathrm{H}$ mode as a function of $B i$ for $\cot \theta=1, \operatorname{Pr}=7, M a=0.1, n=0.8 \ldots \ldots 26$ 


\section{Chapter 1}

\section{Introduction}

Thin-film flow in general refers to flows that are characterized by the fact that the flow domain in one of the dimensions is much smaller than in the other (one or two) dimensions. A typical thin-film flow consists of an expanse of liquid partially bounded by a solid substrate with a (free) surface where the liquid is exposed to another fluid (usually a gas and most often air in applications). Thin liquid films appear in a variety of situations in nature and in engineering applications. Thin-film flow is significant in a broad class of physical applications and attracts the attention of physicists, engineers, and chemists. In the field of engineering, thin liquid layers play an important role in the efficient design and gainful development of units such as thin-film reactors, evaporators, condensers, distillation columns and heat exchangers. Thin film flows are also relevant in various coating applications [1 - 3]. In biological systems, we find thin fluid films such as tear films in the eye, membranes in biophysics, the lining of airways in the lungs of animals, and respiratory flow and transport $[4,5]$. In environmental and geophysical settings, thin flows have been related to geological problems such as gravity currents, lava flows, mud, granular and debris flows, snow avalanches and mudslides $[6,7]$.

The systematic exploration of the flow characteristics of the gravity driven flow of a thin fluid layer down an inclined surface was first carried out by Pyotr Kapitza [8]. Together with his son, Sergey Petrovitch Kapitza, he later conducted the first well-controlled experiment on falling films [9]. Kapitza classified a dimensionless group combining the kinematic surface tension, kinematic viscosity, and gravitational acceleration known as the Kapitza number which is very beneficial in the study of wave growth and instability in falling liquid films. Kapitza predicted that if the Reynolds number was below some critical value which was a function of the Kapitza number, the film flow would be uniformly stable. Though his theoretical estimates apparently matched with his and others' experiments, parts of his analysis were in error. Following Kapitza's groundbreaking theoretical and experimental work, many investigators have contributed to the understanding of the flow characteristics of falling fluid films. The first theoretical investigations were conducted by Benjamin [10] and Yih [11]. Yih performed a linear stability analysis to investigate the long-wave instability of isothermal falling films. His study shows that the basic flat-film flow on an inclined surface is unstable to long-wavelength disturbances if the Reynolds

number is greater than the critical value given by $R e_{c}=\frac{5}{2} \cot \theta$, where $\theta$ is the angle of inclination with 
the horizontal. The Orr-Sommerfeld equation for the evolution of infinitesimal perturbations was used to calculate the critical Reynolds number.

Flow instability of thin non-isothermal liquid films is of importance in engineering applications like facilitating heat transfer and coating application. Falling films on heated planes are subject to instabilities due to shear stress arising from the variation in surface tension due to temperature differences which is referred to as thermocapillarity or the Marangoni effect. This is measured by a dimensionless parameter which is proportional to the variation of surface tension with temperature and is referred to as the Marangoni number. A comprehensive study of interfacial thermocapillary phenomena is given by Nepomnyashchy et al. [12]. Recently the impact of heating on the flow down an inclined plate has been investigated by Kalliadasis et al. [13]. They used a first order integral-boundary-layer approximation to examine the instability of the flow over a uniformly heated inclined plate assuming that surface tension depends linearly on temperature. A second order weighted residual approach was presented by Ruyer-Quil et al. [14] and Scheid et al. [15] to obtain the critical Reynolds number for the onset of instability. The relation between the critical Reynolds number and the Marangoni number has effectively been established by focusing on long-wave perturbations [15 - 17]. Trevelyan et al. [16] considered two different kinds of bottom conditions: constant temperature and constant heat flux, and applied the weighted residual model. While D'Alessio et al. [17] showed that the thermocapillary effect destabilizes the flow over a heated wavy inclined surface.

The influence of temperature dependence in the fluid properties such as density and viscosity have been largely ignored by researches with the justification that the effect is negligible in comparison with that of thermocapillarity for thin layers. However, Ogden et al. [18] have shown that the critical Reynolds number for the onset of instability is unaffected by the variation in the fluid properties only if these are small enough to be of the same order as the wavenumber of the long perturbations that are amplified at the onset of instability. Goussis and Kelly [19] have investigated the effect of variation in viscosity with temperature, however they assumed the temperature at the free surface of the fluid to be prescribed. As a consequence there is no variation in temperature along the surface and the Marangoni effect is eliminated. The same assumption was made by Hwang and Weng [20] who set up a Benney equation and presented a linear and weakly nonlinear stability analysis. On the other hand, Kabova and Kuzenetsov [21] have combined the variation of viscosity with the Marangoni effect, but they only consider the steady-state problem. Recently, Pascal et al. [22] and D'Alessio et al. [23] have included in their investigations of the stability of non-isothermal inclined film flow the Marangoni effect and a linear variation with temperature of all the fluid properties.

The above investigations of conjugated heat and fluid flow have been entirely confined to the Newtonian model. However, in many contexts in industry, engineering and geology, the significant problem deals with how a heated non-Newtonian fluid flows under gravity. The understanding of the physics involved in the flows of such fluids can have direct effects in polymer processing, food stuff processing, design of heat exchangers, wire and fibre coating, ink-jet printing, microfluidics, geological flows in the earth's mantle, hemodynamics and many others. In view of this a lot of interest has been shown towards the study of non-Newtonian flows and hence extensive literature can be found regarding analytic and numerical solutions. The distinguishing feature of a non-Newtonian fluid is a viscoelastic behaviour, or 
if inelastic, i.e. purely viscous, then a nonlinear rheological behaviour relating shear stress and rate of strain. As a consequence then, viscosity is a function of shear rate with the fluid referred to as shear thinning if this is a decreasing function and shear thickening if viscosity increases with shear rate. It turns out that by far the most commonly encountered non-Newtonian behaviour is shear thinning.

Sadiq and Usha [24] analysed the linear instability of a thin layer of viscoelastic liquid flowing down a non-uniformly heated wall. The results show that a non-isothermal viscoelastic film is more stable than the isothermal film and less stable than the corresponding Newtonian film. There have been a number of investigations examining the flow and stability of a viscoelastic fluid film on a vertical/inclined plane, which include the studies in references [24-27].

Regarding purely viscous fluids, it turns out that a power-law relation between shear stress and shear rate is a good fit for the rheological behaviour for many real fluids over a wide range of stresses, and also has the benefit of analytical simplicity. The power-law model has been used effectively in describing the rheological behaviour of polymer solutions and gels. Several investigations of the isothermal flow of a power-law fluid down an incline are reported in references [28 - 31]. Miladinova et al. [28] have considered the influence of the non-Newtonian effect on the stability of a thin film flowing along an inclined plate. Both shear thinning and shear thickening cases are considered. They show that the behaviour of non-Newtonian fluids is similar to that of the Newtonian one, but the amplitude and the shape of the permanent surface waves are both influenced by the non-Newtonian properties of the fluid. Pascal and D'Alessio [31] employ the power-law model, and apply von Karman's momentum integral method to obtain a model for the flow down an incline with an applied wind stress at the surface. By conducting a linear stability analysis they obtain an analytical formula for the relationship between the Reynolds number, the inclination angle and a wind shear parameter which describes the critical conditions for the exponential growth of infinitesimal disturbances.

The stability of the inclined flow of a power-law film in the non-isothermal case has been considered by Sadiq and Usha [32]. At the free surface of the film they implement a thermal insulation condition. A more realistic condition, which is implemented in the recent investigations for the Newtonian case, is Newton's law of cooling which allows for heat transfer from the liquid layer to the ambient gas. The insulation condition leads to an depth-independent equilibrium distribution for the temperature in the liquid layer. Consequently, the formation of waves on the surface does not lead to temperature differences and thus does not generate Marangoni stresses. So, if the surface is assumed to be thermally insulated then the model does not capture the enhancement to the inertial instability due to the Marangoni effect.

Recently, Bernabeu et a. [33] have established a model for lava flow consisting of a viscoplastic fluid with a power-law relation for the viscosity. This investigation does not include the Marangoni effect but a temperature dependence is incorporated into the rheological relation.

In this thesis we study the stability of a power-law fluid film flowing down a heated incline. We implement Newton's law of cooling at the free surface and thus take into account the heat transfer from the liquid layer to the ambient gas above. Our theoretical model also allows for variation in the viscosity of the liquid with temperature. The thesis is organised as follows. In chapter 2 we set up the equations governing the flow. In chapter 3 we obtain an equilibrium solution and apply a linear analysis to study its stability. In chapter 4 we present conclusions drawn from our investigation. 


\section{Chapter 2}

\section{Governing Equations}

\subsection{Dimensional equations}

We consider the two-dimensional flow of a thin liquid film with power-law rheology flowing down an inclined plate heated at a prescribed constant temperature denoted by $T_{w}$. The ambient gas is assumed to remain motionless and at a constant temperature $T_{\infty}<T_{w}$. A rectangular coordinate system is set up as illustrated in Figure 2.1. The position of the free surface of the liquid layer is given by $z=h(x, t)$.

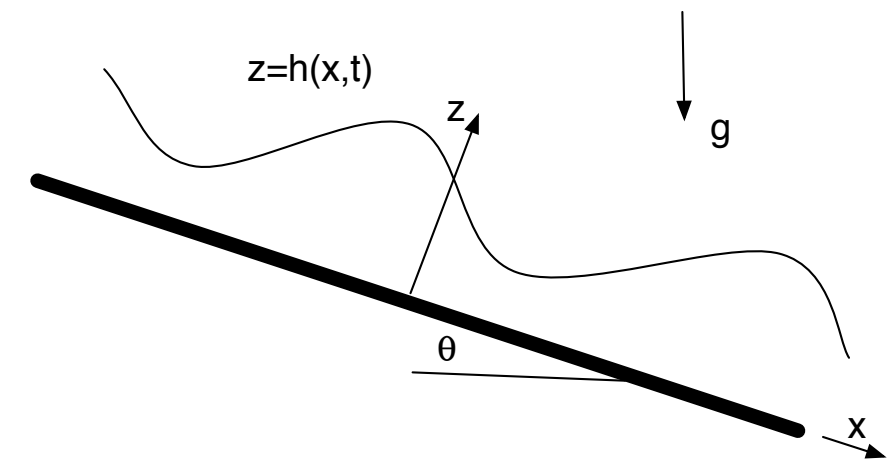

Figure 2.1: Schematic representation of a thin film flowing down an inclined plane.

Equations of motion are obtained from the conservation of mass, momentum and energy. The $x$-momentum equation can be written as

$$
\rho\left(\frac{\partial u}{\partial t}+u \frac{\partial u}{\partial x}+w \frac{\partial u}{\partial z}\right)=-\frac{\partial p}{\partial x}+\frac{\partial \tau_{x x}}{\partial x}+\frac{\partial \tau_{x z}}{\partial z}+\rho g \sin \theta
$$

while the momentum equation in the $z$ direction is given by

$$
\rho\left(\frac{\partial w}{\partial t}+u \frac{\partial w}{\partial x}+w \frac{\partial w}{\partial z}\right)=-\frac{\partial p}{\partial z}+\frac{\partial \tau_{x z}}{\partial x}+\frac{\partial \tau_{z z}}{\partial z}-\rho g \cos \theta,
$$


where $p$ is the pressure and $u$ and $w$ are the streamwise and cross-stream velocity components respectively. The density is given by $\rho$, the gravitational acceleration is denoted by $g$ while $\theta$ represents the angle of inclination of the plate. The components of the deviatoric stress tensor for a power-law fluid are given by $\tau_{x x}=2 \mu_{n} \eta \frac{\partial u}{\partial x}, \tau_{z x}=\tau_{x z}=\mu_{n} \eta\left(\frac{\partial u}{\partial z}+\frac{\partial w}{\partial x}\right)$ and $\tau_{z z}=2 \mu_{n} \eta \frac{\partial w}{\partial z}$, where

$$
\eta=\left[2\left(\left(\frac{\partial u}{\partial x}\right)^{2}+\left(\frac{\partial w}{\partial z}\right)^{2}\right)+\left(\frac{\partial u}{\partial z}+\frac{\partial w}{\partial x}\right)^{2}\right]^{\frac{(n-1)}{2}}
$$

and $\mu_{n}$ and $n$ are the consistency and power-law index respectively. It is worth pointing out here that the classic Newtonian model is a particular case corresponding to $n=1$, with the consistency being the Newtonian viscosity.

In order to incorporate a temperature variation into the rheology of the fluid, we proceed as was done by Bernabeu et al. [33] and assume that the consistency of the fluid is a function of temperature. The usual assumption for the variation in viscosity is an exponential decrease with temperature which is referred to as the Arrhenius formulation. As our focus is a linear stability analysis, we linearize this relation with respect to temperature and assume that the consistency is given by

$$
\mu_{n}=\hat{\mu}_{n}-\hat{\lambda}\left(T-T_{\infty}\right)
$$

where $\hat{\mu}_{n}$ is the value when $T=T_{\infty}$, and $\hat{\lambda}>0$ is a constant control parameter which measures the rate of change with respect to temperature.

From conservation of mass we obtain the continuity equation expressed as

$$
\frac{\partial u}{\partial x}+\frac{\partial w}{\partial z}=0
$$

By applying conservation of energy we obtain the following equation for the temperature of the fluid

$$
\frac{\partial T}{\partial t}+u \frac{\partial T}{\partial x}+w \frac{\partial T}{\partial z}=\chi\left(\frac{\partial^{2} T}{\partial x^{2}}+\frac{\partial^{2} T}{\partial z^{2}}\right)
$$

where $T$ denotes the temperature and $\chi$ is thermal diffusivity.

Dynamical conditions at the free surface of the fluid film are obtained from the continuity of force. Now, the total stress tensor associated with the flow is given by

$$
\vec{s}=-p \vec{I}+\vec{\tau}
$$

where $\vec{\tau}$ denotes the deviatoric stress tensor and $\vec{I}$ is the $2 \times 2$ identity matrix. So, the stress exerted by the flow on the free surface is given by $\vec{s} \cdot \hat{n}$ where

$$
\hat{n}=\frac{1}{\sqrt{1+\left(\frac{\partial h}{\partial x}\right)^{2}}}\left[\begin{array}{c}
-\frac{\partial h}{\partial x} \\
1
\end{array}\right]
$$


is the unit normal vector. We assume that the viscous stress exerted by the ambient gas on the surface is negligible. Therefore, the force of the flow is balanced by the ambient pressure and the effect of surface tension. The total force balance at the surface can then be expressed as

$$
\vec{s} \cdot \hat{n}=-\left(p_{\infty}+2 \sigma K\right) \hat{n}+\overrightarrow{\nabla_{s}} \sigma
$$

where $p_{\infty}$ is the ambient pressure, $\sigma$ is the surface tension, $\nabla_{s}$ is the surface gradient operator which is defined as

$$
\overrightarrow{\nabla_{s}}=\frac{1}{n^{2}}\left[\begin{array}{c}
\frac{\partial}{\partial x}+\frac{\partial h}{\partial x} \frac{\partial}{\partial z} \\
\frac{\partial h}{\partial x} \frac{\partial}{\partial x}+\left(\frac{\partial h}{\partial x}\right)^{2} \frac{\partial}{\partial z}
\end{array}\right]
$$

and $K$ is the mean curvature of the free surface which is given by $K=-\frac{1}{2}\left(\vec{\nabla}_{s} \cdot \widehat{n}\right)$. The normal component of the force balance is obtained by taking the dot product in equation (2.5) with the unit normal vector $\hat{n}$ which yields the following equation

$$
\begin{array}{r}
p=p_{\infty}+\frac{2 \mu_{n} \eta}{1+\left(\frac{\partial h}{\partial x}\right)^{2}}\left[\left(\frac{\partial h}{\partial x}\right)^{2} \frac{\partial u}{\partial x}-\frac{\partial h}{\partial x}\left(\frac{\partial u}{\partial z}+\frac{\partial w}{\partial x}\right)+\frac{\partial w}{\partial z}\right] \\
-\frac{\sigma \frac{\partial^{2} h}{\partial x^{2}}}{\left(1+\left(\frac{\partial h}{\partial x}\right)^{2}\right)^{\frac{3}{2}}} \quad \text { at } z=h(x, t) .
\end{array}
$$

The tangential component of the force balance can be obtained by dotting equation (2.5) with $\hat{t}$, the unit tangent vector, which can be written as $\hat{t}=\frac{1}{\sqrt{1+\left(\frac{\partial h}{\partial x}\right)^{2}}}\left[\begin{array}{c}1 \\ \frac{\partial h}{\partial x}\end{array}\right]$.

We thus obtain

$$
\begin{array}{r}
{\left[\frac{\partial \sigma}{\partial x}+\frac{\partial h}{\partial x}\left(\frac{\partial \sigma}{\partial z}\right)\right] \sqrt{1+\left(\frac{\partial h}{\partial x}\right)^{2}}=\mu_{n} \eta\left[\left(1-\left(\frac{\partial h}{\partial x}\right)^{2}\right)\left(\frac{\partial u}{\partial z}+\frac{\partial w}{\partial x}\right)+2 \frac{\partial h}{\partial x}\left(\frac{\partial w}{\partial z}-\frac{\partial u}{\partial x}\right)\right]} \\
\text { at } z=h(x, t) .
\end{array}
$$

The left-hand side of this equation corresponds to the so-called Marangoni stress which is due to variation in surface tension, and pulls fluid along the surface in the direction of increasing surface tension.

The surface tension is assumed to depend linearly on the temperature and is expressed as

$$
\sigma=\sigma_{\infty}-\sigma_{t}\left(T-T_{\infty}\right)
$$

where $\sigma_{\infty}$ is the surface tension of the fluid at the reference values $T=T_{\infty}$. The parameter $\sigma_{t}$ is defined as

$$
\sigma_{t}=-\frac{\partial \sigma}{\partial T}>0 .
$$


So surface tension decreases with temperature.

A kinematic condition for the free surface of the liquid layer can be derived from the assumption that evaporation is negligible, so fluid particles on the surface must remain on the surface as the fluid flows. The kinematic condition at the surface can be expressed as

$$
w=\frac{D h}{D t}=\frac{\partial h}{\partial t}+\frac{\partial h}{\partial x} u \quad \text { at } \quad z=h(x, t) .
$$

Here $\frac{D h}{D t}$ represents the change in the elevation of the surface.

The relation between the heat flux normal to the surface and the difference in the temperature of the liquid and the ambient gas is expressed through Newton's law of cooling which is given by

$$
-\kappa \nabla T \cdot \widehat{n}=\alpha\left(T-T_{\infty}\right) \quad \text { at } \quad z=h(x, t)
$$

where $\alpha$ is the heat transfer coefficient and $\kappa$ is the thermal conductivity.

At the bottom interface appropriate boundary conditions arise from the requirement of no slip and no penetration and can be written as

$$
u=w=0 \quad \text { at } \quad z=0,
$$

and since the substrate is maintained at temperature $T_{w}$, we have the condition

$$
T=T_{w} \quad \text { at } \quad z=0 .
$$

\subsection{Non-Dimensional equations}

The governing equations can be written in dimensionless form by using an appropriate scaling. For the length scale we choose

$$
H=\left(\frac{\hat{\mu}_{n}}{\rho g \sin \theta}\right)^{\frac{1}{2 n+1}} Q^{\frac{n}{2 n+1}}\left(\frac{2 n+1}{n}\right)^{\frac{n}{2 n+1}} .
$$

This is called the Nusselt thickness, and it corresponds to a steady flow, uniform in the streamwise direction, that would result under isothermal conditions from a prescribed flow rate $Q$. Now, we expect the flow to be destabilized by the amplification of perturbations much longer than the characteristic depth of the fluid layer. We will obtain approximate solutions based on this expectation. In order to be able to identify which terms in the governing equations are negligible, we use a different length scale in the streamwise direction and denote the ratio of $H$ to this scale by $\delta$. To scale the equations we introduce the following transformation

$$
\begin{array}{r}
(x, z)=H\left(\frac{x^{*}}{\delta}, z^{*}\right), h=H h^{*},(u, w)=U\left(u^{*}, \delta w^{*}\right), t=\frac{H}{U \delta} t^{*}, \\
p-p_{\infty}=\rho U^{2} p^{*}, T=T_{\infty}+\Delta T T^{*}
\end{array}
$$


where $U=Q / H$ and $\Delta T=T_{w}-T_{\infty}$.

Scaling the continuity equation gives

$$
\frac{\partial\left(U u^{*}\right)}{\partial\left(L x^{*}\right)}+\frac{\partial\left(U \delta w^{*}\right)}{\partial\left(H z^{*}\right)}=0
$$

Simplifying this equation and dropping the asterisks for notational convenience yields

$$
\frac{\partial u}{\partial x}+\frac{\partial w}{\partial z}=0
$$

Transforming the $x$ - momentum equation gives

$$
\begin{array}{r}
\delta \frac{D u^{*}}{D t^{*}}=-\delta \frac{\partial p^{*}}{\partial x^{*}}+2 \hat{\mu}_{n} \frac{U^{n-2} \delta^{2}}{H^{n} \rho} \frac{\partial}{\partial x^{*}}\left(\left(1-\lambda T^{*}\right) \eta^{*} \frac{\partial u^{*}}{\partial x^{*}}\right) \\
+\hat{\mu}_{n} \frac{U^{n-2}}{H^{n} \rho} \frac{\partial}{\partial z^{*}}\left(\left(1-\lambda T^{*}\right) \eta^{*}\left(\frac{\partial u^{*}}{\partial z^{*}}+\delta^{2} \frac{\partial w *}{\partial x *}\right)\right)+\frac{H}{U^{2}} g \sin \theta,
\end{array}
$$

where

$$
\eta^{*}=\left[2\left(\delta^{2}\left(\frac{\partial u^{*}}{\partial x^{*}}\right)^{2}+\delta^{2}\left(\frac{\partial w^{*}}{\partial z^{*}}\right)^{2}\right)+\left(\frac{\partial u^{*}}{\partial z^{*}}+\delta^{2} \frac{\partial w^{*}}{\partial x^{*}}\right)^{2}\right]^{\frac{n-1}{2}}
$$

and

$$
\lambda=\frac{\hat{\lambda} \Delta T}{\hat{\mu}_{n}} .
$$

Introducing the Reynolds number defined as $R e=\frac{\rho}{\hat{\mu}_{n}} U^{2-n} H^{n}$ and dropping the asterisks we obtain the following scaled $x$-momentum equation

$$
\operatorname{Re} \delta \frac{D u}{D t}=-\operatorname{Re} \delta \frac{\partial p}{\partial x}+2 \delta^{2} \frac{\partial}{\partial x}\left[(1-\lambda T) \eta \frac{\partial u}{\partial x}\right]+\frac{\partial}{\partial z}\left[(1-\lambda T) \eta\left(\frac{\partial u}{\partial z}+\delta^{2} \frac{\partial w}{\partial x}\right)\right]+\left(\frac{2 n+1}{n}\right)^{n}
$$

Scaling the $z$ - momentum equation we get

$$
\begin{array}{r}
\frac{\rho U^{2} \delta}{H} \frac{D w^{*}}{D t^{*}}=-\frac{\rho U^{2} \delta}{H} \frac{\partial p^{*}}{\partial z^{*}} \\
+\hat{\mu}_{n} \frac{U^{n} \delta}{H^{n+1}} \frac{\partial^{*}}{\partial x^{*}}\left(\left(1-\lambda T^{*}\right) \eta^{*}\left(\frac{\partial u^{*}}{\partial z^{*}}+\delta^{2} \frac{\partial w *}{\partial x *}\right)+\hat{\mu}_{n} \frac{U^{n}}{H^{n+1}} \frac{\partial}{\partial z^{*}}\left(\left(1-\lambda T^{*}\right) \eta^{*} \frac{\partial w^{*}}{\partial z^{*}}\right)\right)-\rho g \cos \theta,
\end{array}
$$

which upon simplifying and dropping the asterisks gives

$$
\operatorname{Re} \delta^{2} \frac{D w}{D t}=-\operatorname{Re} \frac{\partial p}{\partial z}+2 \delta^{2} \frac{\partial}{\partial z}\left[(1-\lambda T) \eta \frac{\partial w}{\partial z}\right]+\delta \frac{\partial}{\partial x}\left[(1-\lambda T) \eta\left(\frac{\partial u}{\partial z}+\delta^{2} \frac{\partial w}{\partial x}\right)\right]-\left(\frac{2 n+1}{n}\right)^{n} \cot \theta
$$

Scaling the temperature equation yields

$$
\frac{H U}{\chi} \delta\left(\frac{\partial T *}{\partial t^{*}}+u^{*} \frac{\partial T^{*}}{\partial x^{*}}+w^{*} \frac{\partial T^{*}}{\partial z^{*}}\right)=\delta^{2} \frac{\partial^{2} T^{*}}{\partial x^{* 2}}+\frac{\partial^{2} T^{*}}{\partial z^{* 2}}
$$


which reduces to

$$
\delta \operatorname{RePr}\left(\frac{\partial T}{\partial t}+u \frac{\partial T}{\partial x}+w \frac{\partial T}{\partial z}\right)=\delta^{2} \frac{\partial^{2} T}{\partial x^{2}}+\frac{\partial^{2} T}{\partial z^{2}}
$$

where $\operatorname{Pr}=\frac{\hat{\mu}_{n}}{\rho \chi}\left(\frac{U}{H}\right)^{n-1}$ is the Prandtl number.

Applying the scaling to the continuity of normal stress condition at the free surface yields

$$
\begin{array}{r}
\rho U^{2} p^{*}=\frac{2 \hat{\mu}_{n}}{F^{* 2}} \frac{U^{n}}{H^{n}} \eta^{*}\left(1-\lambda T^{*}\right)\left[\delta^{3}\left(\frac{\partial h^{*}}{\partial x^{*}}\right)^{2} \frac{\partial u^{*}}{\partial x^{*}}+\delta \frac{\partial w^{*}}{\partial z^{*}}-\delta \frac{\partial h^{*}}{\partial x^{*}}\left(\frac{\partial u^{*}}{\partial z^{*}}+\delta^{2} \frac{\partial w^{*}}{\partial x^{*}}\right)\right] \\
-\frac{\delta^{2}}{H F^{* 3}} \frac{\partial^{2} h^{*}}{\partial x^{* 2}}\left(\sigma_{\infty}-\sigma_{t} \Delta T T^{*}\right)
\end{array}
$$

where

$$
F^{*}=\left[1+\delta^{2}\left(\frac{\partial h^{*}}{\partial x^{*}}\right)^{2}\right]^{\frac{1}{2}}
$$

which reduces to

$$
\begin{aligned}
& p=\frac{2 \delta}{R e F^{2}} \eta(1-\lambda T)\left[\delta^{2}\left(\frac{\partial h}{\partial x}\right)^{2} \frac{\partial u}{\partial x}+\frac{\partial w}{\partial z}-\frac{\partial h}{\partial x}\left(\frac{\partial u}{\partial z}+\delta^{2} \frac{\partial w}{\partial x}\right)\right] \\
& -\frac{\delta^{2}}{F^{3}} \frac{\partial^{2} h}{\partial x^{2}}(W e-M T)
\end{aligned}
$$

where $W e=\frac{\sigma_{\infty}}{\rho U^{2} H}$ is the Weber number and is proportional to the surface tension strength and $M=\frac{\sigma_{t} \Delta T}{\rho U^{2} H}$ which measures the variation in surface tension and is sometimes referred to as the film Marangoni number.

Scaling the continuity of tangential stress condition at the free surface leads to

$$
\begin{array}{r}
-\frac{\sigma_{t} \Delta T}{H}\left(\delta \frac{\partial T^{*}}{\partial x^{*}}+\delta \frac{\partial T^{*}}{\partial z^{*}} \frac{\partial h^{*}}{\partial x^{*}}\right)= \\
\hat{\mu}_{n} \frac{U^{n} \eta^{*}}{H^{n}}\left(1-\lambda T^{*}\right)\left[\left(1-\delta^{2}\left(\frac{\partial h^{*}}{\partial x^{*}}\right)^{2}\right)\left(\delta^{2} \frac{\partial w^{*}}{\partial x^{*}}+\frac{\partial u^{*}}{\partial z^{*}}\right)+2 \delta^{2} \frac{\partial h}{\partial x}\left(\frac{\partial w^{*}}{\partial z^{*}}-\frac{\partial u^{*}}{\partial x^{*}}\right)\right]
\end{array}
$$

which reduces to

$$
-M \delta\left(\frac{\partial T}{\partial x}+\frac{\partial T}{\partial z} \frac{\partial h}{\partial x}\right)=\frac{\eta(1-\lambda T)}{R e}\left[\left(1-\delta^{2}\left(\frac{\partial h}{\partial x}\right)^{2}\right)\left(\delta^{2} \frac{\partial w}{\partial x}+\frac{\partial u}{\partial z}\right)-4 \delta^{2} \frac{\partial h}{\partial x} \frac{\partial u}{\partial x}\right] .
$$

Applying the scaling to the Newton's law of cooling yields

$$
\frac{\partial T^{*}}{\partial z^{*}}-\delta^{2} \frac{\partial h^{*}}{\partial x^{*}} \frac{\partial T^{*}}{\partial x^{*}}=-\frac{\alpha H}{K} F^{*} T^{*} \quad \text { at } \quad z^{*}=h^{*}\left(x^{*}, t^{*}\right),
$$


which, upon dropping the asterisks leads to

$$
\frac{\partial T}{\partial z}-\delta^{2} \frac{\partial h}{\partial x} \frac{\partial T}{\partial x}=-B F T \quad \text { at } \quad z=h(x, t)
$$

where $B=\frac{\alpha H}{\kappa}$.

The kinematic condition transforms into

$$
U \delta w^{*}=\frac{\partial\left(H h^{*}\right)}{\partial\left(\frac{H}{U \delta} t^{*}\right)}+\frac{\partial\left(H h^{*}\right)}{\partial\left(L x^{*}\right)}\left(U u^{*}\right) \quad \text { at } \quad z^{*}=h^{*}\left(x^{*}, t^{*}\right),
$$

which, after we drop the asterisks simplifies to

$$
w=\frac{\partial h}{\partial t}+u \frac{\partial h}{\partial x} \quad \text { at } \quad z=h(x, t) .
$$

The remaining boundary conditions at $z=0$ are non-dimensionalized to give

$$
u=0, \quad w=0, \quad T=1 \quad \text { at } \quad z=0 .
$$

Equations (2.16)-(2.24) represent the dimensionless form of the full equations governing the flow. In the next section we simplify these equations by discarding certain terms base on the assumed smallness of the ratio of cross-stream to streamwise length scales. The resulting approximate formulation is known as the long-wave equations.

\subsection{The long-wave approximation}

To obtain the long-wave equations we let $\delta$ tend to zero and discard the $O\left(\delta^{2}\right)$ terms from equations (2.16)-(2.24), while assuming all the other parameters to be $O(1)$, with the exception of the Weber number, which is assumed large enough so that $\delta^{3} W e=O(1)$. This allows us to eliminate the pressure from the problem. Specifically, the $z$-momentum equation and the continuity of normal force condition, equations (2.18) and (2.20), reduce to

$$
\frac{\partial p}{\partial z}=-\left(\frac{2 n+1}{n}\right)^{n} \frac{\cot \theta}{R e}, \quad p=p_{\infty}-\delta^{2} W e \frac{\partial^{2} h}{\partial x^{2}} \quad \text { at } \quad z=h,
$$

which yields the following expression for the pressure

$$
p=p_{\infty}-\delta^{2} W e \frac{\partial^{2} h}{\partial x^{2}}-\left(\frac{2 n+1}{n}\right)^{n} \frac{\cot \theta}{R e}(z-h) .
$$

In obtaining these equations we also discarded the $O(\delta)$ terms since the pressure is multiplied by $\delta$ in the $x$-momentum equation.

Once the negligible terms have been identified and discarded, we rescale the equations with scale $H$ 
for both $x$ and $z$. This can be accomplished by simply setting $\delta=1$ in the current formulation. We thus get the long-wave equations consisting of

$$
\begin{gathered}
\frac{\partial u}{\partial x}+\frac{\partial w}{\partial z}=0 \\
\operatorname{Re}\left(\frac{\partial u}{\partial t}+u \frac{\partial u}{\partial x}+w \frac{\partial u}{\partial z}\right)=\frac{\partial}{\partial z}\left[(1-\lambda T)\left(\frac{\partial u}{\partial z}\right)^{n}\right]-\left(\frac{2 n+1}{n}\right)^{n} \cot \theta \frac{\partial h}{\partial x}+ \\
+\operatorname{ReWe} \frac{\partial^{3} h}{\partial x^{3}}+\left(\frac{2 n+1}{n}\right)^{n} \\
\operatorname{Pr} R e\left(\frac{\partial T}{\partial t}+u \frac{\partial T}{\partial x}+w \frac{\partial T}{\partial z}\right)=\frac{\partial^{2} T}{\partial z^{2}}
\end{gathered}
$$

with the conditions

$$
\begin{gathered}
-M \operatorname{Re}\left(\frac{\partial T}{\partial x}+\frac{\partial T}{\partial z} \frac{\partial h}{\partial x}\right)=(1-\lambda T)\left(\frac{\partial u}{\partial z}\right)^{n}, \quad \text { at } z=h \\
\frac{\partial T}{\partial z}=-B T, \quad \text { at } z=h \\
w=\frac{\partial h}{\partial t}+u \frac{\partial h}{\partial x} \text { at } z=h . \\
u=0, \quad w=0, \quad T=1 \quad \text { at } z=0 .
\end{gathered}
$$

In the next chapter we will employ this model to investigate the stability of the flow. 


\section{Chapter 3}

\section{Linear Stability Analysis}

The problem (2.25) - (2.31) governing a falling power-law fluid film along a heated inclined plate admits a simple solution corresponding to a steady flow uniform in the streamwise direction. In this chapter we obtain this equilibrium solution and investigate its stability by means of a linear analysis.

\subsection{Equilibrium solution}

In order to determine the equilibrium solution we set derivatives with respect to $x$ and $t$ to zero in equations (2.25) - (2.31). We assign the uniform thickness of the liquid layer to be $h=1$ and use the notation $w=w_{s}, u=u_{s}, T=T_{s}$. We thus obtain the following equations for the equilibrium flow

$$
\begin{gathered}
\frac{d w_{s}}{d z}=0 \\
\frac{d}{d z}\left[\left(1-\lambda T_{s}\right)\left(\frac{d u_{s}}{d z}\right)^{n}\right]+\left(\frac{2 n+1}{n}\right)^{n}=0 \\
\frac{d^{2} T_{s}}{d z^{2}}=\operatorname{Pr} \operatorname{Re} w_{s} \frac{d T_{s}}{d z}
\end{gathered}
$$

with the conditions at $z=1$ being

$$
\begin{gathered}
\frac{d u_{s}}{d z}=0 \\
-B T_{s}=\frac{d T_{s}}{d z},
\end{gathered}
$$


and at $z=0$

$$
u_{s}(0)=0, \quad w_{s}(0)=0, \quad T_{s}(0)=1 .
$$

These equations can easily be solved for $T_{s}$ and $w_{s}$ and we obtain

$$
w_{s}(z) \equiv 0
$$

and

$$
T_{s}(z)=1-\left(\frac{B}{B+1}\right) z
$$

In order to obtain a closed-form solution for $u_{s}(z)$ we must resort to an approximation. We integrate equation (3.1) and linearize with respect to $\lambda$ and thus get

$$
\frac{d u_{s}}{d z}=\frac{2 n+1}{n^{2}(B+1)}[n(B+1)+(1+(1-z) B) \lambda](1-z)^{\frac{1}{n}} .
$$

Integrating this and applying the boundary conditions we obtain

$$
\begin{array}{r}
u_{s}=\frac{1}{(B+1)(1+n) n}\left\{B(1-z)^{\frac{1+n}{n}} \lambda n z-B(1-z)^{\frac{1+n}{n}} \lambda n+B(1-z)^{\frac{1+n}{n}} \lambda z\right. \\
-2 B(1-z)^{\frac{1+n}{n}} n^{2}-B(1-z)^{\frac{1+n}{n}} \lambda-B(1-z)^{\frac{1+n}{n}} n+B \lambda n+2 B n^{2}-2(1-z)^{\frac{1+n}{n}} \lambda n \\
\left.-2(1-z)^{\frac{1+n}{n}} n^{2}+B \lambda+n B-(1-z)^{\frac{1+n}{n}} \lambda-(1-z)^{\frac{1+n}{n}} n+2 \lambda n+2 n^{2}+\lambda+n\right\} .
\end{array}
$$

This approximate solution is based on the assumption that $O\left(\lambda^{2}\right)$ terms as $\lambda \rightarrow 0$ are negligibly small.

\subsection{Linear stability theory}

We consider the perturbed equilibrium solution given by

$$
h=1+\zeta(x, t), \quad u=u_{s}(z)+\tilde{u}(x, z, t), \quad w=\tilde{w}(x, z, t), T=T_{s}(z)+\tilde{T}(x, z, t),
$$

where $\zeta, \tilde{u}, \tilde{w}$ and $\tilde{T}$ are the added infinitesimal perturbations.

Introducing the perturbed solution into the governing equations (2.25)-(2.31), then linearizing with respect to the infinitesimal perturbation variables we obtain

$$
\begin{gathered}
\frac{\partial \tilde{u}}{\partial x}+\frac{\partial \tilde{w}}{\partial z}=0 \\
R e\left(\frac{\partial \tilde{u}}{\partial t}+u_{s} \frac{\partial \tilde{u}}{\partial x}+\tilde{w} \frac{d u_{s}}{d z}\right)=-N \cot \theta \frac{\partial \zeta}{\partial x}+\operatorname{ReWe} \frac{\partial^{3} \zeta}{\partial x^{3}}+\frac{d f_{1}}{d z} \tilde{T}+f_{1} \frac{\partial \tilde{T}_{z}}{\partial z}+\frac{d f_{2}}{d z} \frac{\partial \tilde{u}}{\partial z}+f_{2} \frac{\partial^{2} \tilde{u}}{\partial z^{2}}
\end{gathered}
$$




$$
\operatorname{Pr} R e\left(\frac{\partial \tilde{T}}{\partial t}+u_{s} \frac{\partial \tilde{T}}{\partial x}+\tilde{w} \frac{d T_{s}}{d z}\right)=\frac{\partial^{2} \tilde{T}}{\partial z^{2}}
$$

where $f_{1}=-\lambda\left(\frac{d u_{s}}{d z}\right)^{n}, \quad f_{2}=\left(1-\lambda T_{s}\right) n\left(\frac{d u_{s}}{d z}\right)^{n-1}$ and $N=\left(2+\frac{1}{n}\right)^{n}$.

Transferring the boundary conditions at the free surface, i.e. at $z=1+\zeta$, to $z=1$ and linearizing yields the following conditions

$$
\begin{gathered}
-M \operatorname{Re}\left[\frac{\partial \tilde{T}}{\partial x}+\frac{\partial \zeta}{\partial x} \frac{d T_{s}}{d z}\right]=\left(1-\lambda T_{s}\right)\left(\frac{d u_{s}}{d z}\right)^{n-1} n\left(\frac{\partial \tilde{u}}{\partial z}+\zeta \frac{d^{2} u_{s}}{d z^{2}}\right) \quad \text { at } \quad z=1 \\
-B \tilde{T}=\frac{\partial \tilde{T}}{\partial z}+B \frac{d T_{s}}{d z} \zeta \quad \text { at } \quad z=1
\end{gathered}
$$

and

$$
\tilde{w}=\frac{\partial \zeta}{\partial t}+u_{s} \frac{\partial \zeta}{\partial x} \quad \text { at } \quad z=1
$$

At $z=0$ the conditions are

$$
\tilde{u}=0, \quad \tilde{w}=0, \quad \tilde{T}=0
$$

We next employ normal modes into the linearized perturbation equations which are defined as

$$
(\tilde{u}, \tilde{w}, \tilde{T}, \zeta)=(\hat{u}(z), \hat{w}(z), \hat{T}(z), \hat{\zeta}) e^{i k(x-c t)},
$$

where $\hat{\zeta}$ is a constant, $k$ represents the perturbation wavenumber which is a real positive number, $c$ is a complex number whose real is the phase speed of the perturbation, while the product of the imaginary part and $k$ gives the growth rate. Then the linearized perturbation equations (3.5) - (3.11) can be written as

$$
D \hat{w}+i k \hat{u}=0
$$

$$
\begin{aligned}
i k R e\left(u_{s}-c\right) \hat{u}+R e D u_{s} \hat{w}= & -i k\left(N \cot \theta+\operatorname{Re} W e k^{2}\right) \hat{\zeta}+D f_{1} \hat{T}+f_{1} D \hat{T}+D f_{2} D \hat{u}+f_{2} D^{2} \hat{u} \\
& \operatorname{Pr} R e\left[i k\left(u_{s}-c\right) \hat{T}+D T_{s} \hat{w}\right]=D^{2} \hat{T}
\end{aligned}
$$

where $D$ denotes the differentiation with respect to $z$ operator. Applying the normal modes defined above to the interface conditions at $z=1$ gives

$$
-i k M R e\left(\hat{T}+D T_{s} \hat{\zeta}\right)=\left(1-\lambda T_{s}\right)\left(D u_{s}\right)^{n-1} n\left[D \hat{u}+\hat{\zeta} D^{2} u_{s}\right]
$$




$$
\begin{gathered}
-B \hat{T}=D \hat{T}+B D T_{s} \hat{\zeta} \\
\hat{w}=i k\left(u_{s}-c\right) \hat{\zeta} .
\end{gathered}
$$

While the boundary conditions evaluated at $z=0$ will take the following form

$$
\hat{u}(0)=\hat{w}(0)=\hat{T}(0)=0 .
$$

We introduce the stream function, $\psi$, to satisfy the perturbed continuity equation (3.12). The stream function is related to the velocity disturbances $\tilde{u}, \tilde{w}$ and is defined as

$$
\tilde{u}(x, z, t)=\frac{\partial \psi}{\partial z}, \tilde{w}(x, z, t)=-\frac{\partial \psi}{\partial x} .
$$

In terms of the normal modes, $\psi$ can be written as

$$
\psi=\Psi(z) e^{i k(x-c t)} .
$$

Then $\hat{u}$ and $\hat{w}$ are expressed as

$$
\hat{u}(z)=D \Psi, \hat{w}(z)=-i k \Psi .
$$

Consequently, we have the following Orr-Sommerfeld type equations

$$
\begin{gathered}
f_{2} D^{3} \Psi+D f_{2} D^{2} \Psi+i k\left(c-u_{s}\right) \operatorname{Re} D \Psi+i k \operatorname{Re} D u_{s} \Psi \\
+D f_{1} \hat{T}+f_{1} D \hat{T}-i k\left(N \cot \beta+\operatorname{Re} W e k^{2}\right) \hat{\zeta}=0 \\
D^{2} \hat{T}+i k \operatorname{Pr} \operatorname{Re}\left[\left(c-u_{s}\right) \hat{T}+D T_{s} \Psi\right]=0 .
\end{gathered}
$$

The boundary conditions at $z=1$ are

$$
\begin{gathered}
-i k M \operatorname{Re}\left(\hat{T}+\hat{\zeta} D T_{s}\right)=\left(1-\lambda T_{s}\right)\left(D u_{s}\right)^{n-1} n\left[D^{2} \Psi+\hat{\zeta} D^{2} u_{s}\right] \\
-B \hat{T}=D \hat{T}+B D T_{s} \hat{\zeta}
\end{gathered}
$$

and

$$
\Psi+u_{s} \hat{\zeta}=c \hat{\zeta}
$$


While the conditions at $z=0$ are

$$
\hat{T}=\Psi=D \Psi=0
$$

The equations (3.19) - (3.24) constitute an eigenvalue problem with $c$ being the parameter that is to be assigned eigenvalues. For a given set of flow parameters $(\operatorname{Re}, \operatorname{Pr}, B, M, \lambda, n, \theta, W e)$, solving the problem for $c$ provides the growth rate of the perturbation with wavenumber $k$. A positive value of $\Im(c)$ indicates that the perturbation amplitude grows in time, whereas if this quantity is negative then the perturbation is reduced. A set of flow parameters for which $\Im(c)=0$ is referred to as the state of neutral stability for the perturbation with wavenumber $k$, and corresponds to the threshold between stability and instability for this perturbation. Regarding the stability of the flow, if all the perturbations are damped then the flow is stable otherwise, it is unstable.

\subsection{Asymptotic solution}

The solution of the eigenvalue problem (3.19) - (3.24) can be obtained by carrying out an asymptotic analysis as $k \rightarrow 0$. First, we expand the perturbations $\Psi, \hat{T}, \hat{\zeta}$ and the eigenvalue $c$ in powers of $k$ as follows

$$
\begin{aligned}
\Psi & =\psi_{0}(z)+k \psi_{1}(z) \\
\hat{T} & =\hat{T}_{0}(z)+k \hat{T}_{1}(z) \\
\hat{\zeta} & =\hat{\zeta}_{0}+k \hat{\zeta}_{1} \\
c & =c_{0}+k c_{1} .
\end{aligned}
$$

Substituting into the system of equations (3.19) - (3.24), we then have a hierarchy of problems at different orders of $k$. Without loss of generality we can normalize the eigenvalue problem and set $\hat{\zeta}_{0}=1$ and $\hat{\zeta}_{1}=0$.

Collecting the $O(1)$ terms, we obtain

$$
\begin{gathered}
D\left[n\left(1-\lambda T_{s}\right)\left(D u_{s}\right)^{n-1} D^{2} \psi_{0}\right]-D\left[\lambda\left(D u_{s}\right)^{n} \hat{T}_{0}\right]=0 \\
D^{2} \hat{T}_{0}=0
\end{gathered}
$$

with

$$
\begin{gathered}
n\left(1-\lambda T_{s}\right)\left(D u_{s}\right)^{n-1} D^{2} \psi_{0}=-n\left(1-\lambda T_{s}\right)\left(D u_{s}\right)^{n-1} D^{2} u_{s} \quad \text { at } z=1 \\
D \hat{T}_{0}+B \hat{T}_{0}+B D T_{s}=0 \quad \text { at } z=1
\end{gathered}
$$




$$
\psi_{0}+u_{s}=c_{0} \quad \text { at } \quad z=1
$$

and

$$
D \psi_{0}(0)=\psi_{0}(0)=\hat{T}_{0}(0)=0 \text {. }
$$

From equation (3.26) $\hat{T}_{0}$ can be expressed as

$$
\hat{T}_{0}=\bar{A}_{1} z+\bar{A}_{2},
$$

where the constants $\bar{A}_{1}, \bar{A}_{2}$ are determined using the boundary conditions (3.28) and (3.30) giving

$$
\hat{T}_{0}=\frac{B^{2}}{(1+B)^{2}} z
$$

Integrating the ordinary differential equation (3.25) from 1 to $z$, using the boundary condition (3.27) and the fact $D u_{s}(1)=0$ we obtain

$$
n\left(1-\lambda T_{s}\right)\left(D u_{s}\right)^{n-1} D^{2} \psi_{0}-\lambda\left(D u_{s}\right)^{n} \hat{T}_{0}=N
$$

Now linearising with respect to $\lambda$ and applying the boundary conditions (3.30) we obtained an explicit formula for $\psi_{0}(z)$ using the symbolic algebra software Maple. However, the expression is very long and complicated and displaying it does not serve a useful purpose. Substituting the expression into the kinematic condition (3.29) we can solve for $c_{0}$ and obtain

$$
c_{0}=\frac{C_{1}}{C_{2}},
$$

where

$$
\begin{array}{r}
C_{1}=6 n^{3}(B+1)^{2}+\left((4 \lambda+5) B^{2}+(9 \lambda+10) B+6 \lambda+5\right) n^{2}+ \\
\left((4 \lambda+1) B^{2}+(9 \lambda+2) B+5 \lambda+1\right) n+\lambda(B+1)^{2}
\end{array}
$$

and

$$
C_{2}=(B+1)^{2} n^{2}(3 n+1) .
$$

Focusing now on the $O(k)$ terms we obtain the problem

$$
\begin{gathered}
D^{2} \hat{T}_{1}+i \operatorname{Pr} R e\left[\left(c_{0}-u_{s}\right) \hat{T}_{0}+D T_{s} \psi_{0}\right]=0 \\
D \hat{T}_{1}(1)+B \hat{T}_{1}(1)=0
\end{gathered}
$$




$$
\hat{T}_{1}(0)=0,
$$

which has been solved for $\hat{T}_{1}$ using Maple. This solution was then introduced into the equations

$$
\begin{gathered}
D\left[n\left(1-\lambda T_{s}\right)\left(D u_{s}\right)^{n-1} D^{2} \psi_{1}\right]-D\left(\lambda\left(D u_{s}\right)^{n} \hat{T}_{1}\right)=i \operatorname{Re}\left(u_{s}-c_{0}\right) D \psi_{0}-i \operatorname{Re} D u_{s} \psi_{0}+i N \cot \theta \\
n\left(1-\lambda T_{s}\right)\left(D u_{s}\right)^{n-1} D^{2} \psi_{1}=\frac{i M \operatorname{Re} B}{(B+1)^{2}} \quad \text { at } \quad z=1 \\
D \psi_{1}(0)=\psi_{1}(0)=0
\end{gathered}
$$

This problem was also solved using Maple and we obtained a long expression for $\psi_{1}(z)$. We point out that in this process we again linearized with respect to $\lambda$. So the assumption is that $O\left(\lambda^{2}\right)$ and $O\left(k^{2}\right)$ are negligible as $\lambda$ and $k$ tend to zero, but $O(k \lambda)$ is not. Consequently then we are assuming that $k \ll \lambda \ll 1$.

Extracting the $O(k)$ terms from equation (3.23) we obtain $c_{1}=\psi_{1}(1)$. Since $c_{0}$ is real, the state of neutral stability for very long perturbations ( small $k$ ) is given by

$$
\Im\left(c_{1}\right)=0 .
$$

For a Newtonian fluid with constant viscosity it can be shown that infinitely long perturbations are the most unstable [34] and thus relation (3.42) describes the conditions at the threshold of instability for the equilibrium flow, and solving it for Re gives the critical Reynolds number for the onset of instability. We have obtained an explicit formulation for the neutral stability relation (3.42), however it is too long to display here. As an illustration we present a weakly non-Newtonian approximation accurate for power-law index values near $n=1$ given by

$$
R e_{\text {crit }}=\frac{R_{1}}{R_{2}}
$$

where

$$
\begin{gathered}
R_{1}=6(3 n+2)(B+1)^{2}(5 n+2)(5 n+3)(5 n+1)(n+1)^{3} n^{2}(4 n+1)(3 n+1) \cot \theta \\
\left(2 B \lambda n+3 B n^{2}+B \lambda+B n+3 \lambda n+3 n^{2}+\lambda+n\right)(4 n+3), \\
R_{2}=C_{1}+C_{2} \lambda
\end{gathered}
$$


and

$$
\begin{array}{r}
C_{1}=-5376(B+1)\left(10080 B^{2} \ln (3) n\right. \\
+4200 B M \ln (3) n-10080 B^{2} \ln (3)-92844 B^{2} n-4200 B M \ln (3) \\
-44705 n B M+20160 B \ln (3) n+82764 B^{2}+40505 B M-20160 \ln (3) B \\
-185688 B n+10080 n \ln (3)+165528 B-10080 \ln (3)-92844 n+82764)
\end{array}
$$

$$
\begin{array}{r}
C_{2}=-1172247552+1334817792 n-3105636288 B-892433136 B^{3}-2825821872 B^{2} \\
+15052800 B^{2} M \ln (3)+15775032 B^{3} \operatorname{Pr} n+24685848 B^{2} \operatorname{Pr} n-51460992 B \operatorname{Pr} n+142661120 B^{2} M n \\
-124326720 B^{3} \ln (3) n-127608320 B^{2} M+1016759856 B^{3} n-14222712 B^{3} \operatorname{Pr}-22407768 B^{2} \operatorname{Pr} \\
+45977472 B P r+124326720 B^{3} \ln (3)+162570240 \ln (3) \\
+395115840 B^{2} \ln (3)+3220937712 B^{2} n-195175680 B M+433359360 \ln (3) B \\
-162570240 n \ln (3)-15052800 B^{2} M \ln (3) n-22579200 B M \ln (3) n \\
+22579200 B M \ln (3) \\
-395115840 B^{2} \ln (3) n+217754880 n B M-433359360 B \ln (3) n+3538995648 B n .
\end{array}
$$

We also present exact formulations of the neutral stability relation (3.42) for certain particular cases. First of all, in the Newtonian case we obtain

$$
R e_{\text {crit }}=\frac{D_{1}}{D_{2}}
$$

where

$$
D_{1}=1680(B+1)^{2} \cot \theta(3 B \lambda+4 B+4 \lambda+4)
$$

and

$$
\begin{array}{r}
D_{2}=(8064+(231 P r+18501) \lambda) B^{3}+((2240 M+339 P r+58797) \lambda+3360 M+24192) B^{2} \\
+((3360 M-816 P r+64488) \lambda+3360 M+24192) B+24192 \lambda+8064 .
\end{array}
$$

If we set $\lambda=0$ in this result we arrive at

$$
\operatorname{Re}_{\text {crit }}=\frac{10 \cot \theta(B+1)^{2}}{12 B^{2}+(5 M+24) B+12}
$$

which coincides with the result obtained by D'Alessio et al. [17] for the case with constant viscosity. Finally, in the general power-law case under isothermal conditions we have

$$
R e_{c r i t}=\frac{1}{2}\left(\frac{n}{2 n+1}\right)^{2-n}(3 n+2) \cot \theta
$$


which agrees with the formula reported by Fernandez-Nieto et al. [35].

Finally, we point out that the Weber number does not figure in the neutral stability of the flow. This is because the strength of surface tension does not affect very long surface perturbations. It is rather the variation in surface tension, gauged by the parameter $M$, that plays an important role in the Marangoni instability.

\subsection{Interpretation of results}

We first explain the basic physical mechanisms related to the instability of the flow [34]. Non-isothermal inclined film flow is subject to two types of long-wave instabilities: the $\mathrm{H}$ mode and the $\mathrm{S}$ mode. The $\mathrm{H}$ mode refers to the amplification of surface waves due to inertia, and is the only instability mechanism in an isothermal flow. The fluid ahead of a crest on a propagating wave must accelerate when the crest passes over it in order to obtain the faster velocity profile associated with the expanded cross section of the flow. However, fluid inertia resists this adjustment and essentially effectuates a deceleration which causes fluid to accumulate under the crest thus amplifying the amplitude of the wave. At the troughs, inertia accelerates the flow thus pulling fluid away which lowers the troughs which also acts to amplify the wave. Since sufficient inertia is required to destabilize the $\mathrm{H}$ mode, there is a critical Reynolds number such that the $\mathrm{H}$ mode is unstable if this value is exceeded.

If the liquid layer is also heated from below, then a temperature variation is induced along the surface if it is wavy due to differences in distance from the heated bottom. The crests of waves are colder, while the troughs are warmer. As such, surface tension is stronger at the crests and weaker at the troughs generating Marangoni stresses which pull fluid away from the troughs toward the crests thus amplifying the waves. So, thermocapillarity enhances the $\mathrm{H}$ mode and lowers the critical Reynolds number for instability.

Now the S mode refers to the instability that is due entirely to the Marangoni effect when the surface is flat (wavy perturbations being effectively damped due to low levels of inertia). In this case thermocapillarity is generated by perturbations in surface temperature as opposed to differential heating resulting from position relative to the heated bottom. Fluid motion along the surface caused by Marangoni stresses, if sufficiently strong, destabilizes the equilibrium flow. Resisting this action is inertia which works to preserve the equilibrium flow. So, the $\mathrm{S}$ mode is unstable only if the Reynolds number is sufficiently small. The critical value increases with the strength of the thermocapillarity.

The upshot then is that for weak enough thermocapillarity there are two critical Reynolds numbers: one for the $\mathrm{S}$ mode and a larger one for the $\mathrm{H}$ mode. For Reynolds numbers in the interval in between the equilibrium flow is stable while outside the interval it is unstable; due to the $\mathrm{S}$ mode for the smaller values and due to the $\mathrm{H}$ mode for the larger Reynolds numbers. As the level of thermocapillarity is increased, the interval of stable Reynolds numbers shrinks and eventually the two modes merge rendering the flow unstable for all Reynolds numbers.

In order to determine both critical Reynolds numbers, we must redefine two of our current control parameters. It turns out that $B$ and $M$ are implicitly dependent on the Reynolds number. To make 
this explicit we now express them as

$$
B=\left[\csc \theta\left(\frac{2 n+1}{n}\right)^{n}\right]^{\frac{2-n}{n+2}} \operatorname{Re}^{\frac{n}{n+2}} B i
$$

and

$$
M=\left[\csc \theta\left(\frac{2 n+1}{n}\right)^{n}\right]^{\frac{3 n-2}{n+2}} R e^{-\frac{3 n+2}{n+2}} M a,
$$

where

$$
B i=\frac{\alpha}{\kappa}\left(\frac{\mu_{n}}{\rho}\right)^{\frac{2}{n+2}} g^{\frac{n-2}{n+2}}, \quad \text { is the Biot number }
$$

and

$$
M a=\left(\frac{\rho}{\mu_{n}}\right)^{\frac{4}{n+2}} g^{\frac{2-3 n}{n+2}} \sigma_{t} \Delta T, \quad \text { is the Marangoni number. }
$$

The Biot number is the scaled heat transfer coefficient of the free surface. The Marangoni number is proportional to the variation in the surface tension with temperature and measures the level of thermocapillarity for the system. Substituting these expressions for $B$ and $M$ into the neutral stability relation (3.42) results in a much more complicated formula that must be solved for Re numerically. We accomplished this by employing the Matlab subroutine fsolve.

In order to present the results from our investigation we plot the critical Reynolds numbers as a function of the Marangoni number. As it can be seen in Figure 3.1, for example, these curves consist of two branches: The upper which tracks the critical Reynolds number for the $\mathrm{H}$ mode and the lower branch corresponding to the $\mathrm{S}$ mode. The region inside the curve reveals the evolution of the interval of stable Reynolds numbers with $M a$.

We begin by determining how the temperature dependence of the fluid rheology affects the stability of the flow. To accomplish this, in Figures 3.1 and 3.2 we plot the Re crit versus $M a$ curves for different values of $\lambda$. We first consider the Newtonian case in Figure 3.1. Clearly, for a Newtonian fluid increasing $\lambda$ results in a lower viscosity. Also, if we calculate the flow rate in the equilibrium flow we obtain

$$
q_{s}=\int_{0}^{1} u_{s}(z) d z=1+\frac{(3 B+4) \lambda}{4 B+4}
$$

which increases with $\lambda$. Examining the results in Figure 3.1 reveals that $R e_{\text {crit }}$ on the upper branch decreases with $\lambda$, indicating that the $\mathrm{H}$ mode is destabilized. This is most likely a consequence of a faster flow rate which boosts the destabilizing acceleration and deceleration phenomenon brought about by surface waves.

Looking now at the S mode in Figure 3.1 we see that there is no significant effect due to varying $\lambda$, with the exception of cases with greater thermocapillarity. In these cases increasing $\lambda$ has a destabilizing effect. The culprit could possibly be the reduction in viscosity which allows for a stronger fluid motion along the surface generated by the Marangoni stresses.

In Figure 3.2 we consider the effect of $\lambda$ on the stability of the flow for a non-Newtonian fluid which 


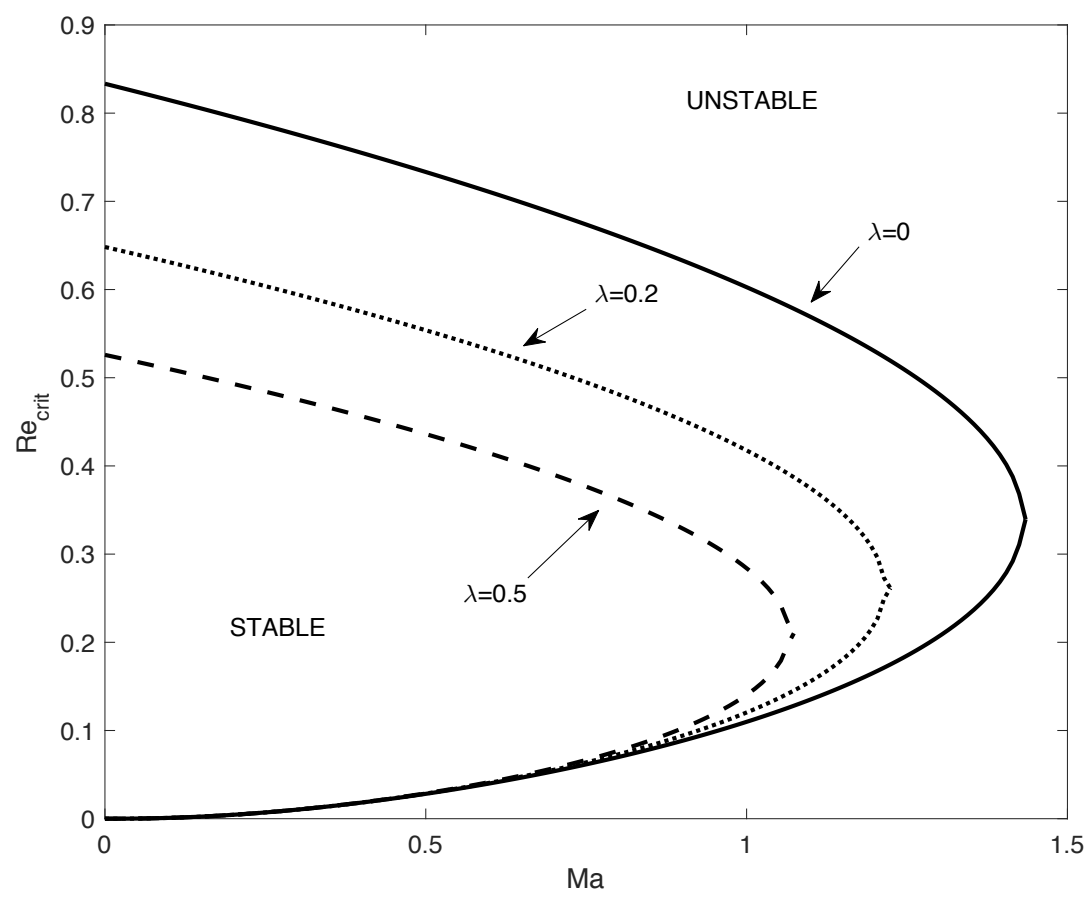

Figure 3.1: $R e_{\text {crit }}$ as a function of $M a$ for $\cot \theta=1, \operatorname{Pr}=7, B i=1, n=1$.

can be obtained from our model by setting the power-law index to $n \neq 1$. It is apparent from these results that a temperature dependent consistency has the same qualitative effect as for a Newtonian fluid. In the non-Newtonian case we do not have a "viscosity" in the Newtonian sense. We can instead think of an "apparent" viscosity defined as $\mu_{a p p}=\mu_{n}\left(\frac{\partial u}{\partial z}\right)^{n-1}$. For the equilibrium flow this is given by

$$
\mu_{\text {app }}=\frac{1}{2 n+1}\left(\frac{2 n+1}{n}\right)^{n}(1-z)^{1-1 / n}\left(n-\frac{1+(1-z) B}{B+1} \lambda\right), \quad 0<z<1,
$$

which, like the Newtonian viscosity, is a decreasing function of $\lambda$. And the flow rate is given by

$$
q_{s}=1+\frac{(2 B+3) n+B+1}{n(3 n+1)(B+1)} \lambda,
$$

which increases with $\lambda$ for all the relevant values of $n$.

We next explore the effect of the non-Newtonian nature of the fluid on the stability of the flow. In Figure 3.3 we display the $R e_{\text {crit }}$ as a function of $M a$ curves for different values of $n$. We include only values less than 1 since this corresponds to most non-Newtonian power-law fluids encountered in applications. It can be seen that the $\mathrm{S}$ mode is stabilized as the fluid is made more non-Newtonian, since the critical Reynolds number decreases. The threshold for the $\mathrm{H}$ mode also decreases, but this means that it is destabilized. However, the rate at which $R e_{c r i t}$ decreases with $M a$ slows down as $n$ is decreased and as a result the interval of stability collapses a larger $M a$ value. In other words, the critical 


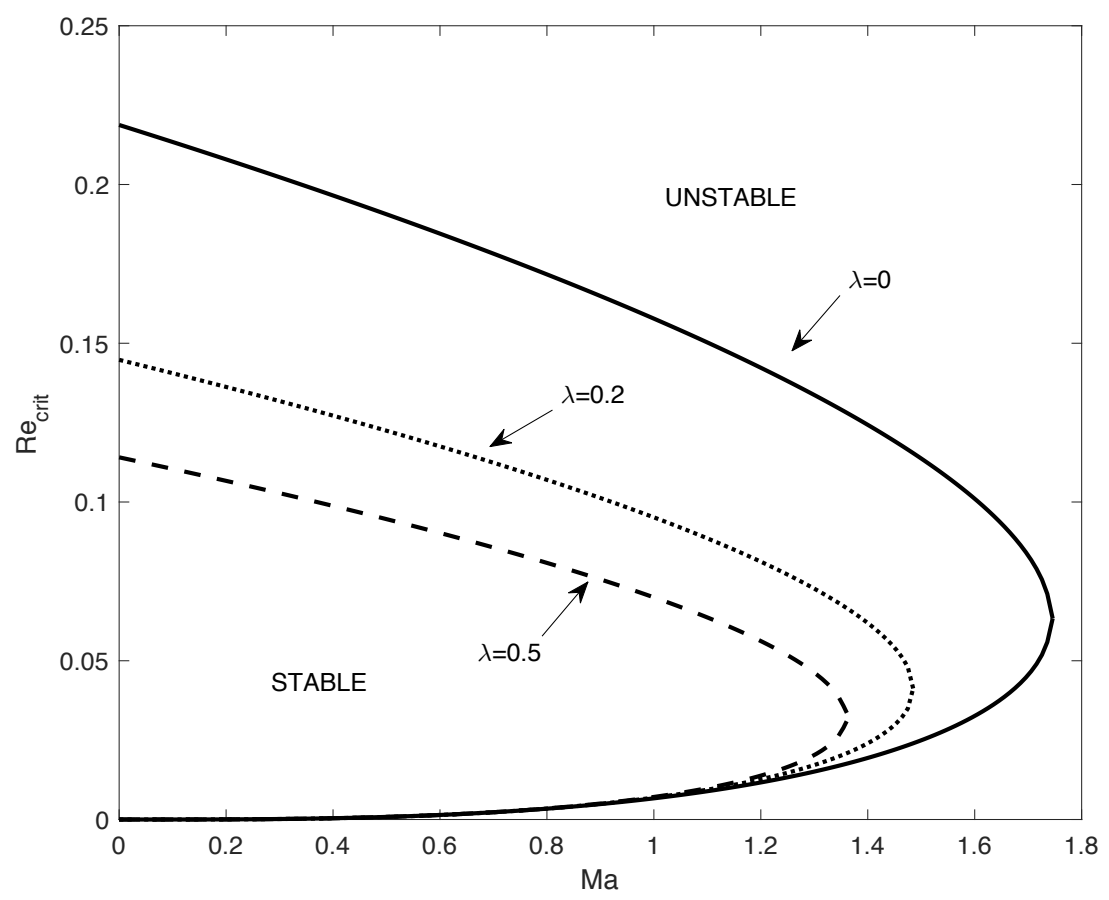

Figure 3.2: $R e_{\text {crit }}$ as a function of $M a$ for $\cot \theta=1, \operatorname{Pr}=7, B i=1, n=0.5$.

$M a$ value for the flow to be rendered unstable for all Reynolds numbers increases as $n$ is decreased. So, for example, if thermocapillarity if sufficiently strong, a Newtonian fluid is unstable for all Reynolds numbers, but for a non-Newtonian fluid there is an interval of Reynolds numbers for which the flow is stable.

We next focus on the effect of the heat transfer across the surface between the liquid and the ambient gas which is measured by the Biot number. We begin by considering the limiting cases $B i=0$ and $B i \rightarrow \infty$. If $B i$ is set to zero then Newton's law of cooling dictates that the heat flux across the surface is prescribed to be zero and consequently the equilibrium flow is isothermal (same temperature throughout). Therefore, even if the surface is wavy its temperature is uniform and so thermocapillarity does not contribute to the amplification of surface waves. At the other extreme we have a similar situation. As $B i \rightarrow \infty$ the condition at the surface approaches that of a prescribed temperature (that of the ambient gas). In this case the surface temperature does not experience any variation in temperature (including perturbations) and thus the Marangoni effect does not occur. Therefore, as we increase $B i$ from zero we expect thermocapillarity to strengthen and the flow to become more unstable. However, this trend must eventually reverse since for large values of $B i$ thermocapillarity becomes weaker.

In Figure 3.4 we consider the Newtonian case and show the critical Reynolds number curves for different values of $B i$ with $\lambda=0$, i.e. no temperature dependence of the consistency of the fluid. In accordance with the expectation described above, we see that for smaller values of $B i$ the region of stability contracts as this parameter is increased, but it begins to expand with $B i$ for sufficiently large 


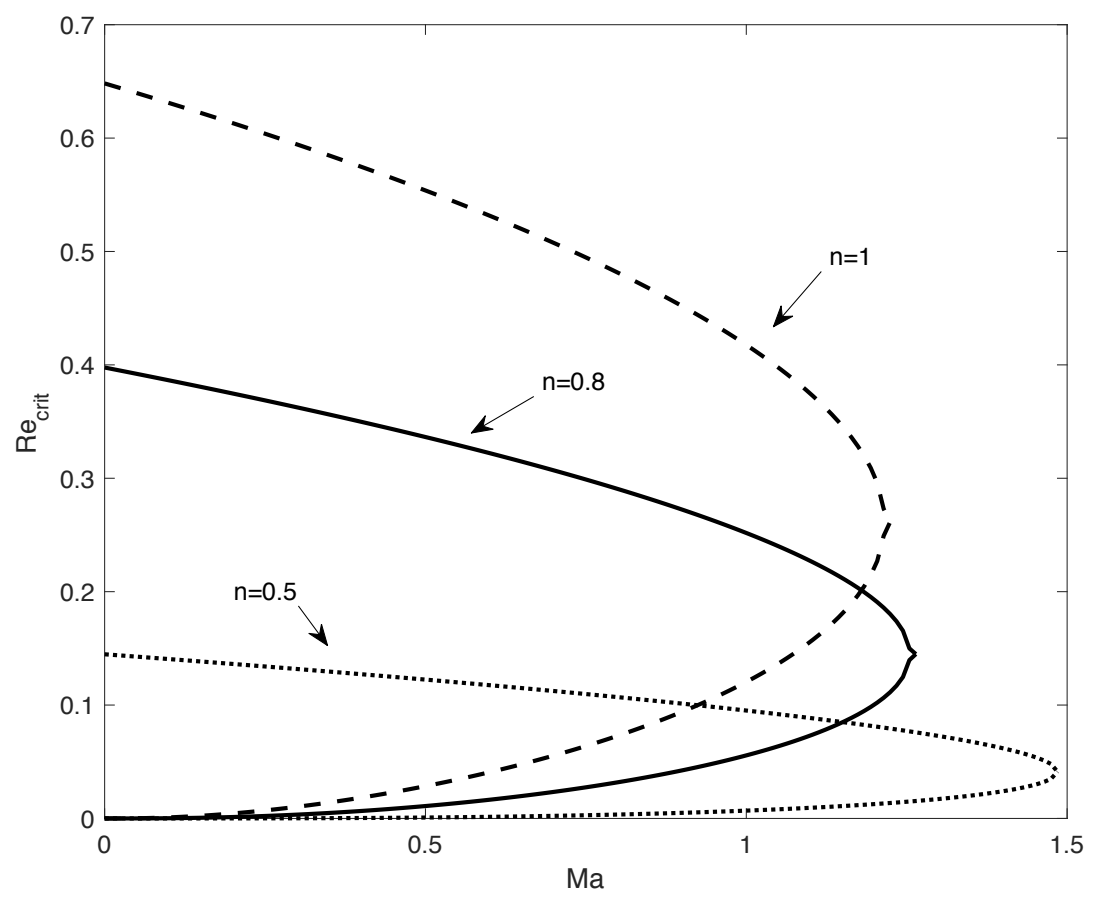

Figure 3.3: $R e_{\text {crit }}$ as a function of $M a$ for $\cot \theta=1, \operatorname{Pr}=7, B i=1, \lambda=0.2$.

values.

Now, if we introduce temperature variation in the viscosity we discover an interesting correlation. Specifically, we find that, for sufficiently small Marangoni numbers, the critical Reynolds number for the $\mathrm{H}$ mode actually increases with $B i$. To illustrate this, in Figure 3.5 we plot $R e_{c r i t}$ as a function of $B i$. To explain this behaviour we point out that with $B i=0$ we have $T_{s}(z) \equiv 1$ (this being the largest possible temperature value), and so the viscosity is reduced by the maximum amount. Now, as explained above, lowering the viscosity destabilizes the flow. So, increasing $B i$ has a stabilizing effect because it relaxes the reduction in viscosity. Yes, it is true that increasing the Biot number from zero also unleashes destabilizing thermocapillarity, however if the surface tension is only weakly dependent on temperature differences (small $M a$ ) the stabilizing effect of increasing the viscosity dominates the Marangoni instability.

This phenomenon is also found for the non-Newtonian case, as it is illustrated in Figure 3.6, where we have set $n=0.8$.

To conclude our analysis of the results we plotted the $R e_{\text {crit }}$ as a function of $M a$ curves for different Prandtl numbers ranging from 0 to 20 . We found these curves to be very close together and almost indistinguishable on the plot, and we do not display the figure here. But we conclude that varying the Prandtl number has little effect on the stability of the flow. 


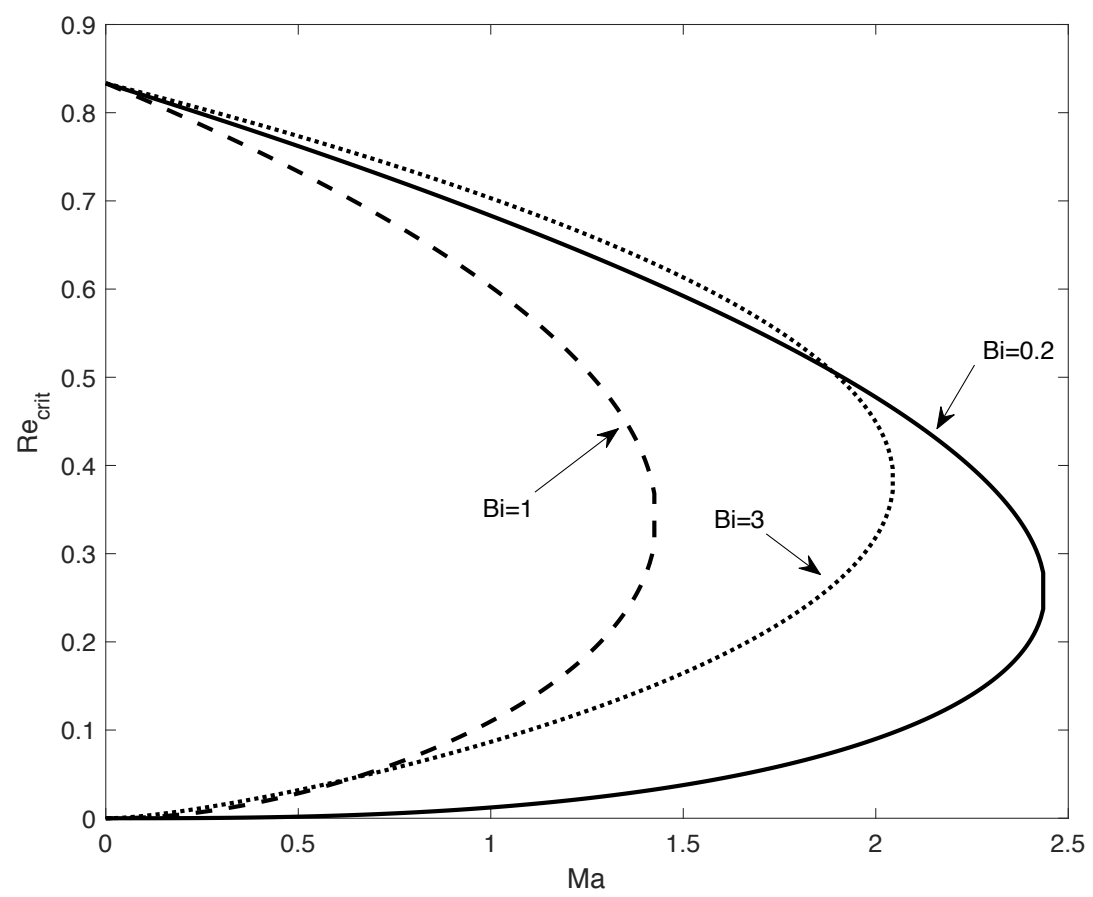

Figure 3.4: $R e_{\text {crit }}$ as a function of $M a$ for $\cot \theta=1, \operatorname{Pr}=7, \lambda=0, n=1$.
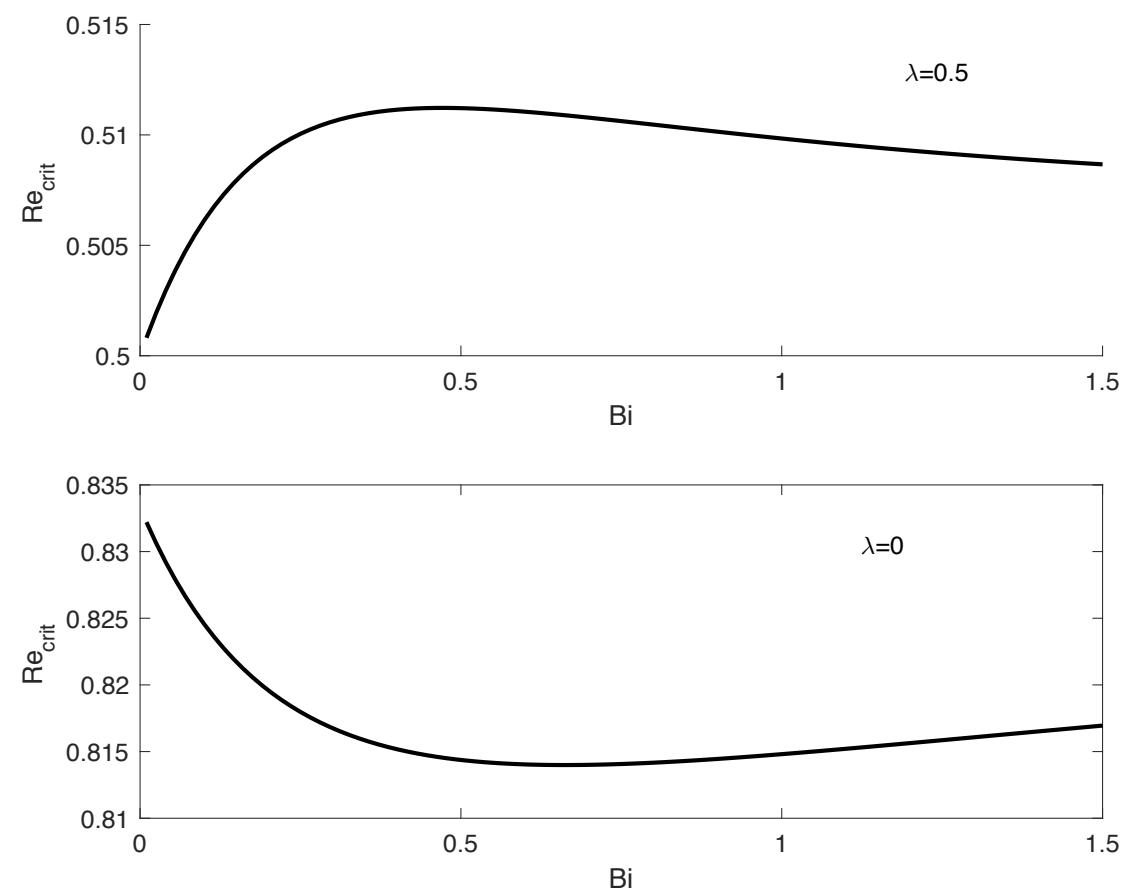

Figure 3.5: Re $e_{\text {crit }}$ for the $\mathrm{H}$ mode as a function of $B i$ for $\cot \theta=1, \operatorname{Pr}=7, M a=0.1, n=1$. 

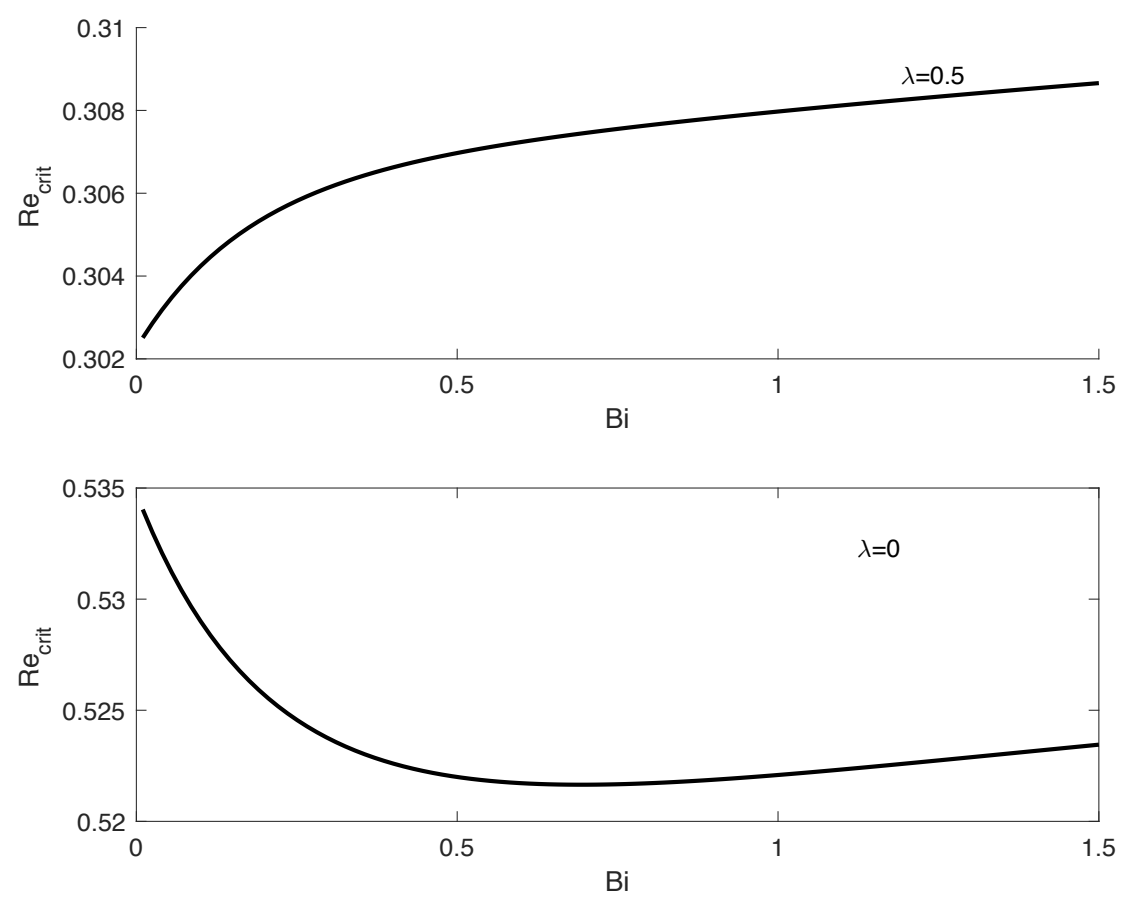

Figure 3.6: $R e_{\text {crit }}$ for the $\mathrm{H}$ mode as a function of $B i$ for $\cot \theta=1, \operatorname{Pr}=7, M a=0.1, n=0.8$. 


\section{Chapter 4}

\section{Conclusions}

In this thesis we derived a mathematical model for the flow of a liquid film down a heated incline. The rheology of the fluid is described by a power-law relation which includes the classic Newtonian fluid, but also allows for non-Newtonian behaviour. The model also accounts for variation with temperature in the properties of the fluid by implementing a temperature dependent formulation for the rheological relation. In addition, the model incorporates the realistic Newton's law of cooling at the surface of the liquid layer, which allows it to capture the enhancement to inertial instability provided by thermocapillarity.

We carried out a linear stability analysis to determine the critical conditions for the onset of longwave instability in the steady flow uniform in the stream wise direction. We accounted for both relevant types of instability. One that is entirely due to thermocapillarity and is associated with slower flows (S mode), and the one that occurs in more rapid flows and consists of the amplification of surface waves due to inertial effects which is intensified by thermocapillarity ( $\mathrm{H}$ mode). The $\mathrm{S}$ mode is stabilized by inertial effects (measured by the Reynolds number), while the opposite is the case for the $\mathrm{H}$ mode. Thermocapillarity raises the level of inertia necessary to stabilize the S mode and lowers the one sufficient to destabilize the $\mathrm{H}$ mode. As thermocapillarity is increased the two thresholds eventually coincide, the two modes are said to "merge", and beyond this level of thermocapillarity the flow is unstable for all Reynolds numbers.

Our investigation has revealed that a non-Newtonian rheology has a stabilizing effect on the $\mathrm{S}$ mode in the sense that a smaller Reynolds number is required to prevent the related amplification of perturbations. Contrarily, the $\mathrm{H}$ mode is destabilized; a smaller Reynolds number is sufficient to amplify perturbations in the position of the free surface. On the other hand, the rate at which the critical Reynolds numbers change with thermocapillarity is lowered by the non-Newtonian aspect of the fluid and the merging of the two modes is postponed. In other words, we can have a flow configuration that is unstable for all Reynolds numbers, but if we replace the fluid by one with the same capillary properties but a stronger non-Newtonian rheological behaviour, we can then obtain a flow that is stable for a specific range of Reynolds numbers.

Regarding the effect of temperature variation in the consistency of the fluid, we found that it destabilizes both modes. Another interesting conclusion pertains to the correlation with the effect of heat 
transfer from the liquid to the ambient gas. In the idealized case of a perfectly insulated free surface, its equilibrium temperature is uniform even if undulations are generated by perturbations. In this case the Marangoni effect is decoupled from the amplification of surface undulations related to inertial effects. As heat is allowed to start flowing across the surface the contribution to the amplification of surface undulations made by thermocapillarity becomes more and more significant and the flow is destabilized. But if the rheological nature of the fluid is affected by temperature the destabilization effect is reversed; increasing the heat transfer from the liquid results in a temperature distribution for which the destabilizing inertial effects themselves are weakened. 


\section{References}

1. Weinstein S. J., Ruschak, K. J., 2004 Coating Flows. Annu. Rev. Fluid Mech 36, 29-53.

2. Myers, T.G., 1996 Surface tension driven thin film flows, in: The Mechanics of Thin Film Coatings, Wiley.

3. Myers, T.G., 1998 Thin films with high surface tension. SIAM Rev 40(3), 441-462.

4. Braun, R., 2012 Dynamics of the tear film, Annu. Rev. Fluid Mech 44 267-297.

5. Grotberg, J.B., 2001 Respiratory fluid mechanics and transport processes, Annu. Rev. Biomed. Eng. 3, 421-457.

6. Ancey, C., 2007 Plasticity and Geophysical Flows: a Review. J. Non-Newt. Fluid Mech 142, $4-35$.

7. Griffiths, R.W., 2000 The Dynamics of Lava Flows. Annu. Rev. Fluid Mech. 32, 477-518.

8. Kapitza, P. L., 1948 Wave flow of thin layers of a viscous fluid: II. Fluid flow in the presence of continuous gas flow and heat transfer. Zh. Eksp. Teor. Fiz. 18, 19-28.

9. Kapitza, P. L. and Kapitza, S. P. 1949 Wave flow of thin layers of a viscous fluid: III. Experimental study of undulatory flow conditions. Zh. Eksp. Teor. Fiz. 19, 105-120.

10. Benjamin, T. B., 1957 Wave formation in laminar flow down an inclined plane.J. Fluid Mech. 2, 554-574.

11. Yih, C.-S., 1963 Stability of liquid flow down an inclined plane. Phys. Fluids 6, 321-334.

12. Nepomnyashchy, A. A., Velarde, M. G., and Colinet, P., 2002, Interfacial Phenomena and Convection, Chapman and Hall, London/CRC, Boca Raton, FL.

13. Kalliadasis, S., Demekhin, E. A., Ruyer-Quil, C., and Velarde, M. G., 2003, Thermocapillary Instability and Wave Formation on a Film Flowing Down a Uniformly Heated Plane, J. Fluid Mech. 492, 303-338.

14. Ruyer-Quil, C., Scheid, B., Kalliadasis, S., Velarde, M., and Zeytounian, R. 2005 Thermocapillary long waves in a liquid film flow. part 1. low-dimensional formulation. J. Fluid Mech. 538, 199-222. 
15. Scheid, B., Ruyer-Quil, C., Kalliadasis, S., Velarde, M., and Zeytounian, R. 2005 Thermocapillary long waves in a liquid film flow. part 2. linear stability and nonlinear waves. J. Fluid Mech. 538, 223-244.

16. Trevelyan, P., Scheid, B.,Ruyer-Quil, C. and Kalliadasis, S. 2007 Heated falling films. J. Fluid Mech. 592, 295-334.

17. D'Alessio, S. J. D., Pascal, J. P., Jasmine, H. A. and Ogden K.A. 2010 Film flow over heated wavy inclined surfaces. J. Fluid Mech. 665, 418-456.

18. Ogden, K. A., D’Alessio, S. J. D. and Pascal, J. P., 2011 Gravity-driven flow over heated, porous, wavy surfaces,Phys. Fluids 23, 122102 .

19. Goussis, D. A. and Kelly, R. E., 1985 Effects of viscosity on the stability of film flow down heated or cooled inclined surfaces: Long-wavelength analysis, Phys. Fluids 28, 3207-3214.

20. Hwang, C.-C. and Weng, C.-I., 1988 Non-linear stability analysis of film flow down a heated or cooled inclined plane with viscosity variation, Int. J. Heat Mass Transfer 31, 1775-1784.

21. Kabova, Y. O. and Kuznetsov, V. V., 2002 Downward flow of a nonisothermal thin liquid film with variable viscosity, J. Appl. Mech. Tech. Phys.43, 895-901.

22. Pascal, J. P., Gonputh, N. and D'Alessio, S. J. D., 2013 Long-wave instability of flow with temperature dependent fluid properties down a heated incline, Int. J. Eng. Sci.70, 73-90.

23. D'Alessio S.J.D., Seth C.J.M.P., and Pascal J.P., The effects of variable fluid properties on thin film stability, Phys. of Fluids 26 122105(2014);

24. Sadiq, I. M. R., and Usha, R., 2005, Linear Instability in a Thin Viscoelastic Liquid Film on an Inclined, Non-Uniformly Heated Wall,Int. J. Eng. Sci., 43,1435-1449.

25. Dandapat, B. S., and Gupta, A. S., 1997, Long Waves on the Surface of a Viscoelastic Fluid Running Down an Inclined Plane, Rheol. Acta, 36, 135-143.

26. Shaqfeh, E. S. G., Larson, R. G., and Fredrickson, G. H., 1989, The Stability of Gravity Driven Viscoelastic Film Flow at Low to Moderate Reynolds Number, J. Non-Newtonian Fluid Mech., 31,87-113.

27. Joo, S. W., 1994, The Stability and Nonlinear Flow Development of a Viscoelastic Draining Film With Shear Thinning, J. Non-Newtonian Fluid Mech., 51, 125-140.

28. Miladinova, S., Lebon, G., and Toshev, E., 2004, Thin-Film Flow of a Power- Law Liquid Falling Down an Inclined Plane, J. Non-Newtonian Fluid Mech., 122, 69-78.

29. Hwang, C. C., Chen, J. L., Wang, J. S., and Lin, J. S., 1994, Linear Stability of Power-Law Liquid Film Flow Down an Inclined Plane, J. Phys. D, 27, 2297-2301. 
30. Perazzo, C. A., and Gratton, J., 2003, Thin Film of Non-Newtonian Fluid on an Incline, Phys. Rev. E, 67, 016307.

31. Pascal, J. P., and D'Alessio, S. J. D., 2007, Instability of a Power-Law Fluid Down an Incline Subjected to Wind Stress, Appl. Math. Model., 31, 1229-1248.

32. Sadiq, I. M. R., and Usha, R., 2009, Long-wave instabilities in a non-Newtonian film on a nonuniformly heated incline, J. Fluid Eng., 131, 031202.

33. Bernabeu, N., Saramito, P. and Smutek, C., 2016, Modelling laval flow advance using shallow-depth approximation for three-dimensional cooling of viscoplastic flows, In: Harris AJL, De Groeve T, Garel F, Carn SA, editors. Detecting, Modelling and responding to effusive eruptions, vol. 426, London: Geological Society, London, Special Publications, 40923.

34. Kalliadasis, S., Ruyer-Quil, C., Scheid, B. and Velarde, M. G. 2012 Falling Liquid Films. (Springer Series on Applied Mathematical Sciences), vol. 176. Springer.

35. Fernandez-Nieto, E.D., Noble, P., Vila, J.-P., 2010, Shallow water equations for non- Newtonian fluids, J. Non-Newtonian Fluid Mech. 165, 712732. 